\title{
DESCRIPCIÓN DEL ESTADO DE SALUD DE LOS TRATAMIENTOS REHABILITADORES EN BASE A IMPLANTES OSEOINTEGRADOS
}

\section{DESCRIPTION OF THE STATE OF HEALTH OF REHABILITATION TREATMENTS BASED ON OSSEOINTEGRATED IMPLANTS}

\author{
Ceballos Rafael ${ }^{1}$, Muñoz Pablo², Steffens Enrique ${ }^{3}$, Trucco Enrique²
}

\section{RESUMEN}

Objetivo: Describir los factores asociados al estado de salud de la rehabilitación sobre implantes oseointegrados (IOI) en los pacientes atendidos en la Central Odontológica de la Primera Zona Naval de la Armada de Chile entre los años 1995 y 2015.

Materiales y métodos: Estudio descriptivo de corte transversal a partir de la recolección de datos obtenidos a través de la evaluación clínica de los pacientes atendidos en la Central Odontológica de la Primera Zona Naval de la Armada de Chile, fichas clínicas y radiografías. Para la clasificación según escala de salud de los implantes se utilizaron los parámetros presentados en el Congreso Internacional de Implantología Oral de Pisa (2007).

Resultados: $65 \mathrm{IOI}$, correspondientes a 30 pacientes, fueron analizados. 63 se encontraron en boca, con un tiempo promedio de 6.9 años de permanencia. El 50,76\% se consideró exitoso, $38,46 \%$ con supervivencia satisfactoria, $3,07 \%$ con supervivencia comprometida y fracasados $7,69 \%$. La asociación entre los cigarrillos diarios y el estado de salud de los IOI presentó un $\mathrm{p}$ valor de 0.040 .

Conclusiones: El fracaso de los implantes tiene un origen multifactorial. La rehabilitación en base a IOI sigue siendo una opción exitosa, predecible y segura para los pacientes.
1. Universidad de Valparaíso, Escuela de Odontología, Valparaíso, Chile.

2. Atención Privada Odontológica, Viña del Mar, Chile.

3. Cirujano Dentista, Corporación Municipal de Valparaíso, Chile.

Correspondencia:

Enrique Steffens Dueñas

Correo electrónico:

enrique.odg@gmail.com

PALABRAS CLAVES:

Implantes oseointegrados; factores de salud; éxito de tratamiento

KEYWORDS:

Osseointegrated Implants; health factors; treatment outcomes 


\section{ABSTRACT}

Objective: To describe health related factors associated with rehabilitation over osseointegrated implants (IOI) of Chilean Navy's First Zone Odontologic Center patients.

Materials and methods: A descriptive crosssectional study was conducted based on the collection of data obtained through the clinical evaluation of Chilean Navy's First Zone Odontologic Center patients, clinical records and radiographs. Parameters presented at the International Congress of Oral Implantology of Pisa (2007) were used for implant health scale classification.

Results: 30 patients were analized. Out of 65 IOI, 63 remained in place, with 6.9 mean years of permanence. $50,76 \%$ IOI were considered successful, $38,46 \%$ were considered to have a satisfactory endurance, $3,07 \%$ had compromised endurance and 7,69\% were unsuccessful. Association between tabaquism and quality of health of IOI presented a $0,040 \mathrm{p}$ value.

Conclusions: Implant failure has a multifactorial origin. IOI-based rehabilitation remains a successful, predictable and safe option for patients.

\section{INTRODUCCIÓN}

La Odontología como ciencia de la salud ha enfocado sus objetivos en rehabilitar a los pacientes de forma integral, devolviendo función, comodidad, estética, fonética y bienestar al sistema estomatognático. La Implantología Oral es una especialidad de la odontología que ha presentado un desarrollo exponencial desde su inicio, hace más de 60 años ${ }^{1}$, lo que ha conllevado a un aumento en la cantidad de especialistas, proveedores $\mathrm{y}$ pacientes para estos tratamientos. Estas nuevas opciones en rehabilitación oral han incentivado la investigación para guiar a los clínicos a establecer procedimientos ideales para sus pacientes. $^{2,5}$

A pesar de ser un área ampliamente investigada, en el plano local, Chile presenta escasas investigaciones en Implantología que incluyan registros de variables relacionadas a la condición sistémica de los pacientes y al estado clínico de la rehabilitación. En este sentido, el conocimiento vigente para estas terapias se ha relacionado en pocas ocasiones con el estado de salud de los pacientes. Estas limitaciones han generado una falta de claridad respecto a las causas de fracaso de este tipo de tratamientos.

Determinar porcentajes de supervivencia y éxito de las rehabilitaciones sobre IOI permitirá distribuir la información para que futuros estudios realicen comparaciones valederas al respecto, de forma que el clínico cuente con suficiente evidencia para guiar sus procedimientos de la forma más efectiva posible.

En la literatura, se han descrito diversos factores de riesgo para el estado de salud de los implantes, divididos en factores locales y sistémicos. Dentro de los factores del implante, existe evidencia de que los diseños más cortos y angostos tienden a generar mayor fracaso en la rehabilitación sobre los que presentan un mayor tamaño. ${ }^{6}$ Dentro de los factores sistémicos se habla de la edad, diabetes, osteoporosis, radioterapia, enfermedades cardiovasculares y tabaquismo como factores de riesgo para el estado de salud de los IOI.

Con este artículo científico se pretende describir de los factores asociados al nivel de salud de la rehabilitación sobre IOI, obteniendo información a partir de los pacientes atendidos en la Central Odontológica de la Primera Zona Naval de la Armada de Chile entre los años 1995 y 2015.

\section{MATERIALES Y MÉTODOS}

Se realizó un estudio descriptivo de corte transversal, diseño que nos permite examinar la relación entre diferentes variables en una población definida en un momento de tiempo determinado.

Para la recolección de datos, se realizaron controles clínicos y radiográficos por parte de un Cirujano Dentista especialista en Implantología Oral, con fichas de registro de información para cada unidad de estudio. La aprobación del proyecto fue responsabilidad del comité de ética de la Universidad de Valparaíso. Además, el especialista obtuvo 
información de los registros clínicos de los pacientes. La medición de la reabsorción ósea radiográfica fue realizada con el programa SIDEXIS XG, a partir de radiografías digitales y análogas digitalizadas. Reuniendo toda esta información se desarrolló una base de datos.

Para la obtención de la muestra, inicialmente se aplicaron los criterios de inclusión al universo, que correspondían a todos los IOI realizados a pacientes atendidos en la especialidad de Implantología Oral de la Central Odontológica de la Primera Zona Naval de la Armada de Chile entre los años 1995 y 2015. Los criterios de inclusión fueron: Pacientes que puedan ser contactados, que se encuentren en la zona, con ficha clínica, implantes endoóseos (IEO) unitarios, IEO rehabilitados, IEO con mínimo un año de carga funcional, implantes con control radiográfico post cirugía. Los criterios de exclusión fueron: Pacientes no dispuestos a asistir al control clínico-radiográfico, pacientes que no pueden recibir radiación (embarazadas).

Las variables cualitativas dicotómicas del estudio fueron: Sexo, resistencia a la insulina, diabetes, hipertensión arterial, hipotiroidismo, dislipidemia, supervivencia IOI (presente o ausente en boca), dolor (presente o ausente), profundidad de sondaje (mayor valor $>7 \mathrm{~mm}$ o $<7 \mathrm{~mm}$ ) y movilidad (presente o ausente).

Las variables cualitativas policotómicas fueron: Persistencia tabaco (fumador, exfumador hace más de 1 año, no fumador), longitud IOI (cortos 6-9 mm, medios 10-12 $\mathrm{mm}$, largos 13-18 mm), diámetro IOI (base pequeña o angosta $<3.75 \mathrm{~mm}$, regular $=3.75$ $\mathrm{mm}$ y ancha $>3.75 \mathrm{~mm}$ ), tratamientos complementarios (ninguna terapia adicional, regeneración ósea guiada (ROG), injerto óseo en bloque), exudado (presente, historia de exudado, ausente) y reabsorción ósea promedio $(<2 \mathrm{~mm}, 2$ a $4 \mathrm{~mm},>4 \mathrm{~mm}$ y mayor a $1 / 2$ del IOI). Las variables cuantitativas discretas del estudio fueron: Edad (medida en años), cantidad de cigarrillos diarios y tiempo del implante en boca (medida en años). Los datos cualitativos son presentados como frecuencias o porcentajes, mientras que los datos cuantitativos se presentan como medias y desviación estándar.

Para la clasificación del estado de salud de los implantes, se utilizó la tabla presentada en el Congreso Internacional de Implantología Oral de Pisa (2007). Según estos criterios, se lleva a cabo la escala de salud de los IOI según cuatro categorías: Exitoso, supervivencia satisfactoria, supervivencia comprometida o fracaso. (Tabla I)

Con el fin de evitar o disminuir sesgo o error sistemático, las medidas fueron realizadas por un solo examinador, especialista en Implantología Oral.

La técnica de muestreo correspondió a una del

Tabla I: Escala de salud de los implantes dentales, ICOI Pisa, 2007.

\begin{tabular}{|c|c|c|c|c|c|}
\hline & $\begin{array}{c}\text { Dolor o } \\
\text { Sensibilidad }\end{array}$ & Movilidad & Pérdida ósea & Exudado & Otros \\
\hline Exitoso & Ausente & Ausente & $<2 \mathrm{~mm}$ & $\begin{array}{c}\text { Sin } \\
\text { antecedentes }\end{array}$ & - \\
\hline $\begin{array}{l}\text { Supervivencia } \\
\text { satisfactoria }\end{array}$ & Ausente & Ausente & $2-4 \mathrm{~mm}$ & $\begin{array}{c}\text { Sin } \\
\text { antecedentes }\end{array}$ & - \\
\hline $\begin{array}{l}\text { Supervivencia } \\
\text { comprometida }\end{array}$ & $\begin{array}{c}\text { Posible } \\
\text { sensibilidad }\end{array}$ & Ausente & $>4 \mathrm{~mm}$ & $\begin{array}{c}\text { Posible } \\
\text { antecedente }\end{array}$ & $\begin{array}{l}\text { Profundidad } \\
\text { de sondaje } \\
>7 \mathrm{~mm}\end{array}$ \\
\hline Fracaso & Presente & Presente & $1 / 2$ del IOI & $\begin{array}{c}\text { Con } \\
\text { antecedente }\end{array}$ & Pérdida IOI \\
\hline
\end{tabular}


tipo no probabilístico por asociación casual.

Con respecto a los métodos estadísticos, se utilizaron los softwares Microsoft Excel 365 y Stata 14, para la confección de la base de datos y tablas. Para las variables cualitativas, se aplicó el test $\chi^{2}$ cuadrado de Pearson con un IC de $95 \%$, para determinar dependencia o no de las variables. Se determinó un $\mathrm{p}$ valor $<\mathrm{a}$ 0.05 como estadísticamente significativo.

\section{RESULTADOS}

A partir de la aplicación de los criterios de inclusión y exclusión, se obtuvo una población de 93 implantes dentales. Para definir la muestra, se citó a evaluación a todos los participantes, con un total de 65 IOI efectivamente controlados y debidamente documentados en la historia clínica, quedando fuera de este estudio los implantes de pacientes que no asistieron a las citaciones, por no poder ser contactados o por no encontrarse dentro de la zona. Finalmente, estos 65 IOI, correspondientes a 30 pacientes, fueron los utilizados para el análisis de esta investigación.

Del total de pacientes, 20 eran masculinos y 10 femeninos, con un promedio de edad de 57 +10 años. Con respecto a las condiciones sistémicas, se encontraron 4 pacientes con resistencia a la insulina, 1 paciente diabético, 9 pacientes con hipertensión arterial, 3 pacientes con hipotiroidismo y 5 pacientes con dislipidemia. Con respecto al tabaquismo, según la cantidad de cigarrillos diarios y la persistencia del hábito, se encontraron 18 pacientes no fumadores (60\% del total), 2 exfumadores $(66,6 \%$ del total), 9 fumadores leves ( $<6$ cigarrillos diarios, $30 \%$ del total) y 1 fumador moderado (entre 6 y 15 cigarrillos diarios, 3,33\% del total).

Estado de salud IOI: Para el estado de salud de los IOI, se utilizó la escala de salud descrita en la sección de materiales y métodos. Los parámetros descritos incluían: Presencia del implante, dolor (al momento de la evaluación), profundidad de sondaje, presencia actual o pasada de exudado, reabsorción ósea promedio en comparación con radiografía inicial y movilidad del implante. (Tabla II)

Tabla II: Distribución de las variables del estado de salud de los implantes según su frecuencia y porcentaje.

\begin{tabular}{|c|c|c|}
\hline Variables clínicas & Frecuencia & $\%$ \\
\hline \multicolumn{3}{|l|}{ Presencia implante } \\
\hline Presente & 63 & 96,92 \\
\hline Ausente & 2 & 3,07 \\
\hline \multicolumn{3}{|l|}{ Dolor } \\
\hline Presente & 1 & 1,58 \\
\hline Ausente & 62 & 98,41 \\
\hline \multicolumn{3}{|l|}{$\begin{array}{l}\text { Profundidad de sondaje } \\
\text { (mayor valor) }\end{array}$} \\
\hline$>7 \mathrm{~mm}$ & 2 & 3,17 \\
\hline$<7 \mathrm{~mm}$ & 61 & 96,82 \\
\hline \multicolumn{3}{|l|}{ Exudado } \\
\hline Presente & 0 & 0 \\
\hline Historia de exudado & 3 & 4,76 \\
\hline Ausente & 60 & 95,23 \\
\hline \multicolumn{3}{|l|}{$\begin{array}{l}\text { Reabsorción ósea } \\
\text { promedio }\end{array}$} \\
\hline$<2 \mathrm{~mm}$ & 41 & 65,07 \\
\hline $2-4 \mathrm{~mm}$ & 19 & 30,15 \\
\hline $\begin{array}{c}>4 \mathrm{~mm}(\text { menos de la } 1 / 2 \\
\text { del IOI }\end{array}$ & 3 & 4,76 \\
\hline Mayor de $1 / 2$ del IOI & 0 & 0 \\
\hline \multicolumn{3}{|l|}{ Movilidad } \\
\hline Presente & 5 & 7,93 \\
\hline Ausente & 58 & 92,06 \\
\hline
\end{tabular}

En base a estos resultados, se clasificaron en 33 implantes exitosos (50,76\% del total), 25 implantes con supervivencia satisfactoria $(38,46 \%$ del total), 2 implantes con supervivencia comprometida $(3,07 \%$ del total) y 5 implantes fracasados $(7,69 \%$ del total).

Supervivencia del implante: De los 65 IOI evaluados, 63 se encontraron en boca a la hora de la evaluación, correspondientes al 96,92\% del total de IOI.

Tiempo de IOI en boca: El promedio de tiempo en boca de los IOI fue de 9,6 + 6 años. Cabe destacar, que 12 implantes no fueron registrados dentro de este criterio debido a que no se encontraba información que especificara 
la cantidad de años desde su instalación.

Longitud: Se obtuvo un total de 4 implantes cortos $(6-9 \mathrm{~mm}), 20$ implantes $(10-12 \mathrm{~mm})$ medios y 41 (13-18 mm) implantes largos.

Diámetro: Se obtuvo un total de 11 implantes de base pequeña o angosta $(<3,75 \mathrm{~mm}), 8$ implantes de base regular $(3,75 \mathrm{~mm})$ y 46 implantes de base ancha (> 3,75 $\mathrm{mm})$.

Tratamientos complementarios: Al $66,15 \%$ del total de IOI no se les realizó ninguna terapia adicional, a $18,46 \%$ se les realizó regeneración ósea guiada previa a su colocación y $15,38 \%$ del total de IOI requirieron un injerto óseo en bloque para su inserción.

La longitud, el diámetro y los tratamientos complementarios resultaron ser variables independientes al estado de salud del IOI en el análisis estadístico de $\chi^{2}$.

Para la asociación entre condiciones sistémicas y estado de salud de los implantes, todas las variables exceptuando el hipotiroidismo, resultaron ser independientes. Con un $\mathrm{p}$ valor menor a 0.05 , podemos afirmar que existe evidencia estadísticamente significativa de que la presencia de hipotiroidismo controlado y el estado de salud de los implantes son variables dependientes. (Tabla III)

Para la asociación entre los cigarrillos diarios y el estado de salud de los implantes expuestos y no expuestos a este hábito, también se obtuvo un $\mathrm{p}$ valor menor a 0.05 , indicando que existe evidencia estadísticamente significativa de que el aumento en el consumo de cigarrillos en su medición diaria y el estado de salud de implantes son variables dependientes. (Tabla IV)

\section{DISCUSIÓN}

Tasa de supervivencia: A partir de las evaluaciones clínicas, se registró una tasa de supervivencia de un 96,92\%: 63 de los 65 implantes incluidos en el estudio se encontraban en boca a la hora de realizar el examen. Estos índices se condicen con lo encontrado en la literatura: Moraschini y cols. realizaron una revisión sistemática en donde se incluyeron 23 estudios de evaluación de implantes dentales, de un mínimo de 10 años de seguimiento. De esto se obtuvo una tasa de supervivencia promedio de $94,6 \%$ de los implantes. ${ }^{7,8}$ A través de esto, podemos reafirmar la predictibilidad y seguridad del

Tabla III: Asociación entre hipotiroidismo y estado de salud de los implantes.

\begin{tabular}{|c|c|c|c|c|c|}
\hline Hipotiroidismo & Exitoso & S. Satisfactoria & S. Comprometida & Fracaso & Total \\
\hline Ausente & 28 & 22 & 2 & 1 & $\mathbf{5 3}$ \\
\hline $\begin{array}{c}\text { Presente } \\
\text { controlado) }\end{array}$ & 5 & 3 & 0 & 4 & $\mathbf{1 2}$ \\
\hline Total & $\mathbf{3 3}$ & $\mathbf{2 5}$ & $\mathbf{2}$ & $\mathbf{5}$ & $\mathbf{6 5}$ \\
\hline \multicolumn{7}{|c|}{ Pearson Chi $2=13,9650,003$} \\
\end{tabular}

Tabla IV: Asociación entre cigarrillos diarios y estado de salud de los implantes.

\begin{tabular}{|c|c|c|c|c|c|}
\hline $\begin{array}{c}\text { Cigarrillos } \\
\text { diarios }\end{array}$ & Exitoso & S. Satisfactoria & S. Comprometida & Fracaso & Total \\
\hline 0 & 25 & 18 & 1 & 3 & $\mathbf{4 7}$ \\
\hline 1 & 3 & 0 & 0 & 0 & $\mathbf{3}$ \\
\hline 2 & 1 & 2 & 1 & 0 & $\mathbf{4}$ \\
\hline 4 & 2 & 0 & 0 & 2 & $\mathbf{4}$ \\
\hline 5 & 2 & 3 & 0 & 0 & $\mathbf{5}$ \\
\hline 7 & 0 & 2 & 0 & 0 & $\mathbf{2}$ \\
\hline Total & $\mathbf{3 3}$ & $\mathbf{2 5}$ & $\mathbf{2}$ & $\mathbf{5}$ & $\mathbf{6 5}$ \\
\hline \multicolumn{7}{|l}{} \\
\hline
\end{tabular}


tratamiento rehabilitador sobre IOI.

Relación entre factores de riesgo y estado salud IOI: Se encontró una dependencia entre la cantidad de cigarrillos diarios y el estado de salud de los implantes. Esta relación ha sido ampliamente estudiada. Dentro de los estudios previos se ha descrito que los efectos locales y sistémicos producidos por el tabaco generan alteraciones en el metabolismo óseo, perjudicando el mecanismo de cicatrización involucrado en la oseointegración del IOI. ${ }^{9,10}$ En este sentido, también se ha establecido que el hábito de fumar aumenta las tasas de fracaso en la rehabilitación sobre implantes y la pérdida ósea marginal. ${ }^{11}$ A partir de la literatura, podemos determinar que existe plausibilidad biológica para la relación de dependencia entre tabaquismo y estado de salud de IOI encontrada en nuestro estudio.

Con respecto a la dependencia entre el hipotiroidismo y el estado de salud de los implantes, a pesar de que se ha descrito una alteración en el proceso de remodelado óseo en pacientes con hipotiroidismo producto de la disminución de la disponibilidad de hormonas tiroideas, los autores Alsaadi y Attard concluyeron que no existe asociación entre esta condición y el fracaso de los implantes dentales. ${ }^{12,13}$ Luego, pese a obtener un resultado de dependencia entre el estado de salud del implante y el hipotiroidismo, es posible considerar que esto sea producto de factores locales y no de la condición sistémica propiamente tal.

La evaluación de las variables se desarrolló no sólo a partir del éxito o fracaso de los implantes, sino que también incluyó criterios que permitieron conformar la escala de salud diseñada en Pisa 2007. De tales criterios se obtuvo un $51 \%$ de éxito, un $38 \%$ de supervivencia satisfactoria, un $3 \%$ de supervivencia comprometida y un $8 \%$ de fracasos. Cabe destacar que el fracaso no está asociado sólo a la pérdida del implante, sino que también a criterio que indican la necesidad de extracción del mismo. Al relacionar con la literatura, no se encontraron estudios que utilicen la escala de salud de forma completa $\mathrm{y} / \mathrm{o}$ con grupos heterogéneos de pacientes, sino que fue empleada con muestras con factores de riesgo específicos, lo que impide la comparación de tales resultados. Pese a esto, fue posible determinar una mayoría de tratamientos exitosos, lo que se corresponde con el carácter seguro, predecible y exitoso ampliamente analizado en la literatura con respecto a la rehabilitación sobre IOI.

En cuanto a las limitaciones, el tamaño de la muestra fue insuficiente, ya sea por los pacientes que no quisieron asistir a los controles, como quienes cambiaron su lugar de residencia, imposibilitando su asistencia o contacto. Se suma a esto los pacientes que no cumplieron los criterios de inclusión, con registros físicos incompletos o perdidos.

Dado el tipo de estudio, solo se describen los datos y no se puede establecer una relación fehaciente entre los factores de riesgo descritos.

Finalmente, las unidades de estudio fueron estudiadas en una población heterogénea pero perteneciente a un sistema de salud diferenciado del resto del país.

Se sugiere aumentar el tamaño y representatividad de la muestra, para obtener resultados más concluyentes.

Extender este tipo de estudio a otro tipo de poblaciones, pertenecientes a otros sistemas de servicio de salud.

Estimular la realización de estudios analíticos que relacionen los factores presentados con los diferentes niveles de salud de los IOI.

\section{CONCLUSIÓN}

Evidencia estadísticamente significativa para determinar el carácter dependiente del tabaquismo y el hipotiroidismo para el estado de salud de los implantes.

Los factores incluidos dentro de este estudio fueron comparados con los descritos en la literatura.

El fracaso de los implantes tiene un origen multifactorial.

La rehabilitación oral en base a IOI sigue 
siendo una opción exitosa, predecible y segura para los pacientes.

\section{REFERENCIAS}

[1] Lindh T, Gunne J, Tillberg A, Molin M. A meta-analysis of implants in partial edentulism. Clin Oral Implants Res. 1998 Apr;9(2):80-90.

[2] Vasak, C., Fiederer, R. \& Watzek, G. Current state of training for implant dentistry in Europe: a questionnaire-based survey. Clinical Oral Implants Research 2007;18: 1320. DOI: 10.1111/j.1600-0501.2007.01499.x

[3] Watzek, G. Oral implants - quo vadis? The International Journal of Oral \& Maxillofacial Implants 2006; 21: 831-832.

[4] Pommer B, Zechner W, Watzak G, Ulm C, Watzek G, Tepper G. Progress and trends in patients' mindset on dental implants. I: level of information, sources of information and need for patient information. Clinical Oral Implants Research. 2010;22(2):223-229. DOI: 10.1111/j.1600-0501.2010.02035.x

[5] Allen, P.F. \& McMillan, A. Food selection and perceptions of chewing ability following provision of implant and conventional prostheses in complete denture wearers. Clinical Oral Implants Research 2002;13: 320-326.

[6] Esposito M, Hirsch JM, Lekholm U, Thomsen P. Biological factors contributing to failures of osseointegrated oral implants. (I) Success criteria and epidemiology. Eur $\mathrm{J}$ Oral Sci 1998; 106: 527-551. 6

[7] Moraschini V, Poubel L, Ferreira V, Barboza E. Evaluation of survival and success rates of dental implants reported in longitudinal studies with a follow-up period of at least 10 years: A systematic review. Int $J$ Oral Maxillofac Surg [Internet]. 2015;44(3):377388. DOI: 10.1016/j.ijom.2014.10.023

[8] Pjetursson BE, Thoma D, Jung R, Zwahlen M, Zembic A. A systematic review of the survival and complication rates of implantsupported fixed dental prostheses (FDPs) after a mean observation period of at least 5 years. Clin Oral Impl Res. 2012;23(Suppl 6):22-38. DOI: 10.1111/j.1600-0501.2012.02546.x

[9] Pereira ML, Carvalho JC, Peres F, Fernandes MH. Simultaneous effects of nicotine, acrolein, and acetaldehyde on osteogenicinduced bone marrow cells cultured on plasma-sprayed titanium implants. Int $\mathbf{J}$ Oral Maxillofac Implants. United States; 2010;25(1):112-22.

[10] Lindquist LW, Carlsson GE, Jemt T (1997) Association between marginal bone loss around osseointegrated mandibular implants and smoking habits: a 10-year follow-up study. J Dent Res 76: 1667-167. DOI: 10.1177/00220345970760100801

[11] Chrcanovic BR, Albrektsson T, Wennerberg A. Smoking and dental implants: A systematic review and meta-analysis. J Dent. England; 2015 May;43(5):487-98. DOI: 10.1016/j.jdent.2015.03.003

[12] Alsaadi G, Quirynen M, Komárek A, van Steenberghe D. Impact of local and systemic factors on the incidence of oral implant failures, up to abutment connection. $\mathrm{J}$ Clin Periodontol, 2007; 34: 610-617. DOI: 10.1111/j.1600-051X.2007.01077.x

[13] Attard NJ, Zarb GA. A study of dental implants in medically treated hypothyroid patients. Clin Implant Dent Relat Res, 2002; 4: 220-231.

\section{CÓMO CITAR ESTE ARTÍCULO}

Ceballos R, Muñoz P, Steffens E, Trucco E. Descripción del estado de salud de los tratamientos rehabilitadores en base a implantes oseointegrados. Appli. Sci. Dent. 2020; 1(1): 40-46. DOI: 10.22370/asd.2020.1.1.2112 